\title{
Non-wandering sets of the powers of maps of the interval
}

\author{
ETHAN M. COVEN' AND ZBIGNIEW NITECKI \\ Department of Mathematics, Wesleyan University, Middletown, Conn.; and \\ Department of Mathematics, Tufts University, Medford, Mass.
}

(Received 6 June 1980 and revised 8 December 1980)

\begin{abstract}
We show that, for maps of the interval, the non-wandering set of the map coincides with the non-wandering set of each of its odd powers, while the nonwandering set of any of its even powers can be strictly smaller.
\end{abstract}

\section{Introduction}

In the study of the dynamics of a continuous map $g: X \rightarrow X$ of a metrizable space to itself, a central role is played by the various recursive properties of the points of $X$. One such property is periodicity: $x$ is periodic if $g^{n}(x)=x$ for some $n \geq 1$. A weaker property is that of being non-wandering: $x$ is non-wandering if for every neighbourhood $U$ of $x, g^{n}(U) \cap U \neq \varnothing$ for some $n \geq 1$; equivalently, $x$ is non-wandering if there exist $x_{i} \rightarrow x$ and $k_{i} \rightarrow \infty$ such that $g^{k_{i}}\left(x_{i}\right) \rightarrow x$. Intermediate recursive properties (which we shall not consider here) include almost periodicity and recurrence.

This paper investigates the non-wandering sets of the powers (under composition) of a map $g$, particularly when the underlying space is a compact interval. Throughout this paper, $f: I \rightarrow I$ will be understood to denote such a 'map of the interval'.

Let $P=P(g)$ denote the set of periodic points and $\Omega=\Omega(g)$ the set of nonwandering points of $g$. In general, $\Omega$ is closed, invariant and, if $X$ is compact, non-empty. By contrast, $P$ need be only invariant, although for maps of the interval, $P$ is non-empty.

In general, $\bar{P} \subseteq \Omega$. Equality holds $C^{r}$-generically $(1 \leq r \leq \infty)$ for maps of the interval [15]. When equality fails for a map of the interval, the difference set $\Omega-\bar{P}$ is small. It is nowhere dense and has invariant measure zero [4] and, for piecewise monotone maps, it consists entirely of isolated points [11].

It is clear that $P(g)=P\left(g^{n}\right)$ for all $n \geq 1$. (The corresponding results hold for almost periodicity and recurrence as well [6] [7].) It is well-known, however, that $\Omega(g)=\Omega\left(g^{n}\right)$ does not hold in general. In fact, it can fail even for diffeomorphisms of surfaces [12]. If $\Omega(f) \neq \Omega\left(f^{n}\right)$ for some $n \geq 2$, then this phenomenon must be exhibited by some point in the pathological set $\Omega-\bar{P}$.

We shall prove:

MAIN THEOREM. For maps of the interval, $\Omega(f)=\Omega\left(f^{n}\right)$ whenever $n$ is odd.

It follows from the main theorem that if $n=2^{k} m$ with $m$ odd, then $\Omega\left(f^{n}\right)=\Omega\left(f^{2 k}\right)$.

† Address for correspondence: Ethan M. Coven, Department of Mathematics, Wesleyan University, Middletown, Conn. 06457, USA. 
Thus all the possible sets $\Omega\left(f^{n}\right)$ appear in the nested sequence

$$
\Omega(f) \supseteq \Omega\left(f^{2}\right) \supseteq \Omega\left(f^{4}\right) \supseteq \cdots .
$$

We shall indicate how any sequence of equalities and strict containments in (*) may be realized.

The main theorem is an immediate corollary of the following two results.

THEOREM 1. If $x \in \Omega$ has an infinite orbit, then $x \in \Omega\left(f^{n}\right)$ for all $n \geq 1$.

THEOREM 3. If $x \in \Omega$ has a finite orbit, then $x \in \Omega\left(f^{n}\right)$ for all odd $n \geq 1$.

For light maps, i.e. maps which do not collapse any interval to a point, we establish the following result.

THEOREM 2. For light maps, if $x \in \Omega\left(f^{N}\right)$ and the $f$-orbit of $x$ contains a fixed point of $f^{N}$, then $x \in \Omega\left(f^{n}\right)$ for all $n \geq 1$.

We include a number of examples, sprinkled throughout the paper, which illuminate some of the high points of the theory, as well as showing its necessity. Taken together, these examples illustrate the delicate nature of the set $\Omega-\bar{P}$.

We also include a section at the end which indicates some of the obstructions to extending our results to spaces of dimension greater than one.

\section{An example}

In [15], L.-S. Young constructed an example of a map of the interval with $\bar{P} \neq \Omega$. It can be checked that in Young's example $\Omega(f)=\Omega\left(f^{n}\right)$ for all $n \geq 1$. (In fact, it is a simple application of theorem 2 of this paper.)

The following example is similar to Young's, but in this case $\Omega(f)=\Omega\left(f^{n}\right)$ for all odd $n \geq 1$ and $\Omega\left(f^{2}\right)=\Omega\left(f^{4}\right)=\Omega\left(f^{6}\right)=\cdots$ is a proper subset of $\Omega(f)$.

Let $f:[0,1] \rightarrow[0,1]$ be differentiable and let $0<x_{0} \leqslant p<q<c<1$ satisfy (figure 1):

(1) $f\left(x_{0}\right)=p, f(p)=q, f(q)=p, f(c)=x_{0}$.

(2) $f^{\prime}\left(x_{0}\right)>0, f^{\prime}(p)<-1, f^{\prime}(q)<-1, f^{\prime}(c)=0$.

(3) $f\left[0, x_{0}\right] \subseteq\left[x_{0}, p\right], f\left[x_{0}, p\right] \subseteq[p, 1], f[p, q]=[p, q], f[q, c]=\left[x_{0}, p\right], f[c, 1] \subseteq$ $\left[x_{0}, p\right]$.

(4) For every neighbourhood $V$ of $q, c \in f^{2 n}(V)$ for some $n \geq 0$.

It is clear that there exist arbitrarily smooth, in fact analytic, maps satisfying (1)-(4).

(1.1) Proposition. With notation as above, $x_{0} \in \Omega\left(f^{n}\right)$ if and only if $n$ is odd.

Proof. We consider neighbourhoods of $x_{0}$ of the form $U=\left[x_{0}-\varepsilon, x_{0}+\varepsilon\right]$. It suffices to show that for $\varepsilon>0$ small enough, $f^{2 n}(U) \cap U=\varnothing$ for all $n \geq 1$ and (since every odd number has arbitrarily large odd multiples) $f^{2 n+1}(U) \cap U \neq \varnothing$ for sufficiently large $n$.

Let $\varepsilon>0$ be small enough so that

(5) $f\left(x_{0}-\varepsilon\right)>x_{0}+\varepsilon$.

(6) $f\left[x_{0}-\varepsilon, x_{0}\right]=\left[f\left(x_{0}-\varepsilon\right), p\right]$.

(7) $f\left[x_{0}, x_{0}+\varepsilon\right] \subseteq[p, q]$. 


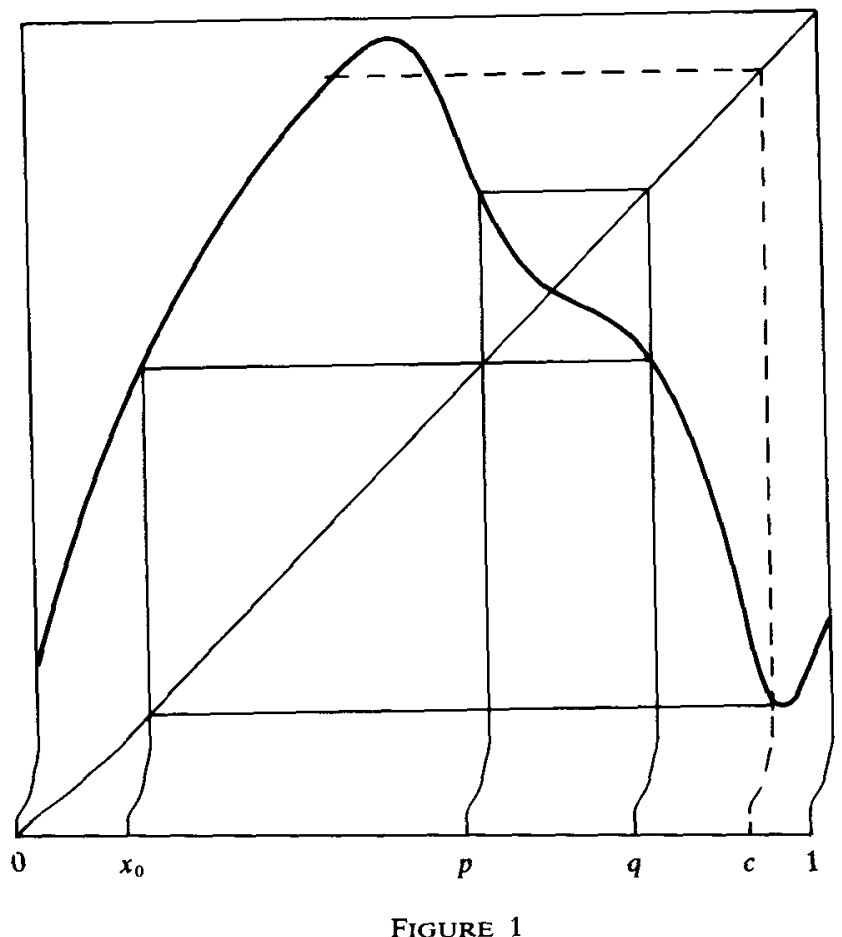

Figure 1

Since $f^{2}(U) \subseteq[p, 1]$ and $f^{2}[p, 1] \subseteq[p, 1]$, the statement about the even powers of $f$ follows. On the other hand, since $f^{2}(U)$ is a neighbourhood of $q$, (4) implies that $c \in f^{2 n}(U)$ for sufficiently large $n$. For any such $n, x_{0} \in f^{2 n+1}(U) \cap U$.

We shall prove the main theorem by showing that, in some sense, the behaviour of any point in $\Omega(f)$ but not in $\Omega\left(f^{n}\right)$ for some $n \geq 2$ must mimic the behaviour of the point $x_{0}$ in this example.

\section{Some technical facts}

In this section we set down some useful facts about non-wandering points and maps of the interval. The most important of these is (2.6) which asserts that, as in the example of $\S 1$, if $x \in \Omega$, then for every neighbourhood $U$ of $x$, there exists $n \geq 1$ such that $f^{n}(U) \cap U$ is not only non-empty, but actually contains $x$.

The first two facts are immediate consequences of the definitions, and hold for all continuous maps.

(2.1) If $m$ divides $n$, then $\Omega\left(g^{n}\right) \subseteq \Omega\left(g^{m}\right)$.

(2.2) $\mathrm{cl}[P(g)] \subseteq \Omega\left(g^{n}\right)$ for all $n \geq 1$.

Thus if $\Omega(f) \neq \Omega\left(f^{n}\right)$ for some $n \geq 2$, this behaviour must be exhibited by some point in $\Omega-\bar{P}$.

The next two facts are immediate consequences of the intermediate value theorem. 
(2.3) If $J \subseteq I$ is a closed interval and either $f(J) \subseteq J$ or $f(J) \supseteq J$, then $f$ has a fixed point in $J$.

(2.4) If $J \subseteq I$ is an interval containing no periodic points, then for every $n \geq 1$, either $f^{n}(x)<x$ for all $x \in J$ or $f^{n}(x)>x$ for all $x \in J$.

(2.5) Lemma. Let $J \subseteq I$ be an interval, open in $I$, such that for every $n \geq 1$, no endpoint of $J$ (other than endpoints of $I$ ) lies in int $\left[f^{n}(J)\right]$. Then either $f^{n}(J) \cap J=\varnothing$ for all $n \geq 1$, or there exists $N \geq 1$ such that $f^{n}(J) \cap J \neq \varnothing$ only if $n$ is a multiple of $N$, and in this case $\bar{J} \supseteq f^{N}(\bar{J}) \supseteq f^{2 N}(\bar{J}) \supseteq \cdots$.

Proof. Let $N$ be the least positive integer such that $f^{N}(J) \cap J \neq \varnothing$, if such exists. Then $f^{N}(\bar{J}) \subseteq \bar{J}$ and tile sequence of containments follows.

If $1 \leq r \leq N-1$, then $f^{k N+r}(J) \subseteq \operatorname{cl}\left[f^{r}(J)\right]$ which fails to meet $J$ since $J$ is open in $I$.

The following result was proved for piecewise monotone maps by Young [15].

(2.6) LEMMA. If $x \in \Omega(f)$, then there exist $x_{i} \rightarrow x$ and $k_{i} \rightarrow \infty$ such that $f^{k_{i}}\left(x_{i}\right)=x$.

Proof. It suffices to show that there exist $x_{i} \rightarrow x$ and $k_{i} \geq 1$ such that $f^{k_{i}}\left(x_{i}\right)=x$. For if $\left\{k_{i}\right\}$ were bounded, then $x$ would be periodic and the conclusion of the lemma would hold with $x_{i}^{\prime}=x$ and $k_{i}^{\prime}=n i$ where $n$ is the period of $x$. Thus we need to show that $x \in \operatorname{cl}\left[\bigcup_{n \geq 1} f^{-n}(x)\right]$.

Suppose that $x \notin \operatorname{cl}\left[\bigcup f^{-n}(x)\right]$. Let $J$ be the component of $I-\operatorname{cl}\left[\bigcup f^{-n}(x)\right]$ containing $x$. Then $J$ satisfies the hypotheses of (2.5). Let $N$ be the integer given by (2.5). Then $x \in \Omega\left(f^{N}\right)$, for if $U \subseteq J$ is a neighbourhood of $x$, then $f^{n}(U) \cap U \neq \varnothing$ for some $n \geq 1$, which by $(2.5)$ is a multiple of $N$.

We claim that $x \notin f^{3 N}(\bar{J})$. For if $x=f^{3 N}(y)$ where $y \in \bar{J}$, then the three points $y$, $f^{N}(y), f^{2 N}(y)$ are distinct points of $\bar{J}$ and hence at least one of them belongs to $J$. But all three points are in $\mathrm{cl}\left[\bigcup f^{-n}(x)\right]$, and so the claim follows.

Since $x \notin f^{3 N}(\bar{J})$, there is a neighbourhood $V \subseteq J$ of $x$ such that $V \cap f^{3 N}(\bar{J})=\varnothing$. But then $f^{k N}(V) \cap V=\varnothing$ for all $k \geq 3$. This implies that $x \notin \Omega\left(f^{N}\right)$, a contradiction.

(2.7) LeMMA. No endpoint of $I$ is in $\Omega-\bar{P}$.

Proof. Let $I=[a, b]$. If $a \in \Omega-\bar{P}$, then there is an interval $J=[a, a+\varepsilon]$ such that $J \cap P=\varnothing$. Since $f^{n}(a)>a$ for all $n \geq 1$, it follows from $(2.4)$ that $f^{n}(x)>x$ for all $x \in J$ and all $n \geq 1$. Then by (2.6), $a \notin \Omega$.

The proof that $b \notin \Omega-\bar{P}$ is similar.

\section{Theorem 1}

In this section, we prove

THEOREM 1. If $x \in \Omega$ has an infinite orbit, then $x \in \Omega\left(f^{n}\right)$ for all $n \geq 1$.

We shall find it convenient throughout the remainder of this paper to use the notion of one-sided 'neighbourhoods'. An $L$-neighbourhood of $x$ is a subinterval of $I$ 
of the form $V_{L}=[x-\varepsilon, x]$ for some $\varepsilon>0 . R$-neighbourhoods are defined analogously: $V_{R}=[x, x+\varepsilon]$.

If $x$ is an endpoint of $I$, then the appropriate one-sided neighbourhoods form a neighbourhood base at $x$. If $x$ is not an endpoint, then every closed, connected neighbourhood $V$ of $x$ is the union of two non-degenerate one-sided neighbourhoods: $V=V_{L} \cup V_{R}$.

To prove theorem 1, suppose $x \in \Omega$ has an infinite orbit and let $n \geq 2$. We show that $x \in \Omega\left(f^{n}\right)$. By (2.2), if $x \in \bar{P}$, then $x \in \Omega\left(f^{n}\right)$. Suppose then that $x \notin \bar{P}$ and let $V$ be a closed, connected neighbourhood of $x$. We may assume that $V \cap P=\varnothing$.

By (2.6), there exist $x_{i} \rightarrow x$ and $m_{i} \rightarrow \infty$ such that $f^{m_{i}}\left(x_{i}\right)=x$. Without loss of generality, $V_{L}$ contains infinitely many $x_{i}$. It suffices to show that the set of 'return times' of $V_{L}$ to $x$, i.e. $\left\{m \geq 1 \mid x \in f^{m}\left(V_{L}\right)\right\}$, contains multiples of $n$.

To do this, let $R$ denote the set of residue classes modulo $n$ which include infinitely many return times of $V_{L}$ to $x$.

$$
R=\left\{[r] \mid x \in f^{m}\left(V_{L}\right) \text { for infinitely many } m \in[r]\right\} .
$$

We show that $[0] \in R$. Since $R$ is finite, by shrinking $V$ and taking subsequences, we may assume that if $[r] \in R$, then there exist $x_{i} \in V_{L}$ and $m_{i} \in[r]$ with $x_{i} \rightarrow x, m_{i} \rightarrow \infty$ and $f^{m_{i}}\left(x_{i}\right)=x$.

(3.1) Proposition. If $f^{i n}(x) \in \operatorname{int}\left[f^{k n+r}\left(V_{L}\right)\right]$ for some $j, k, r \geq 0$, then $[r+s] \in R$ for every $[s] \in R$.

Proof. There exists an $L$-neighbourhood $W_{L} \subseteq V_{L}$ of $x$ such that $f^{\text {in }}\left(W_{L}\right) \subseteq f^{k n+r}\left(V_{L}\right)$.

Let $[s] \in R$. There are infinitely many $y \in W_{L}$ and $m \in[s]$ with $f^{m}(y)=x$. For any such $y$ and $m=l n+s$ with $l>j$, we have $x=f^{l n+s}(y) \in f^{(l-j+k) n+(r+s)}\left(V_{L}\right)$. Therefore $[r+s] \in R$.

(3.2) Proposition. [0] $\in R$.

Proof. Since there are only finitely many residue classes, $R \neq \varnothing$. Thus it suffices to show that the hypotheses of (3.1) are satisfied for every $[r] \in R$.

Let $[r] \in R$. Choose $k_{1}<k_{2}<k_{3}$ so that $x \in V_{i}=f^{k_{i} n+r}\left(V_{L}\right)$ for $i=1,2,3$. If $x \in V_{i}^{\circ}$ for some $i$, then the hypotheses of (3.1) are satisfied with $j=0$ and $k=k_{i}$. Hence we assume that $x$ is an endpoint of each $V_{i}$. Since $f^{k_{i} n+r}\left(z_{i}\right)=x>z_{i}$ for some $z_{i} \in V_{L}$, it follows from (2.4) that $f^{k_{i} n+r}(x)>x$. Therefore $x$ is the left endpoint of each $V_{i}$.

Now consider the three points $x_{i}=f^{\left(k_{i}-k_{1}\right) n}(x) \in V_{i}$. Since the orbit of $x$ is infinite, these points are distinct and hence one of them lies between the other two. Since $x_{1}=x$ is the left endpoint of both $V_{2}$ and $V_{3}$, either $x_{1}<x_{2}<x_{3}$ or $x_{1}<x_{3}<x_{2}$. In the first case, $x_{2} \in V_{3}^{\circ}$ and the hypotheses of (3.1) are satisfied with $j=k_{2}-k_{1}$ and $k=k_{3}$. In the second case, $x_{3} \in V_{2}^{\circ}$ and the hypotheses of (3.1) are satisfied with $j=k_{3}-k_{1}$ and $k=k_{2}$.

This completes the proof of theorem 1 .

\section{Denjoy maps of the interval}

One might well try to prove theorem 1 by establishing the following conjecture: if $x \in \Omega$ has an infinite orbit, then $x \in \bar{P}$. In this section, we construct an example which 
shows this conjecture to be false. The fact that the example, unlike the example in $\S 1$, is relatively complicated, illustrates the delicate nature of the set $\Omega-\bar{P}$. The reader interested primarily in the development of the theory required to prove the main theorem is advised to skip this section for now, and return to it after $\S 10$.

The existence of smooth maps of the interval with 'homtervals' has aroused some interest of late [8][9]. A homterval is a non-degenerate, closed subinterval $J$ which is (i) not contained in the domain of attraction of any periodic orbit, (ii) for which $\left\{f^{n}(J) \mid n \geq 0\right\}$ is a collection of pairwise disjoint, non-degenerate, closed intervals, and (iii) such that $f^{n} \mid J$ is a homeomorphism for every $n \geq 1$. With a little extra care in the construction of our example, we can make it $C^{1}$ (but not $C^{2}$ ) and have homtervals.

Our examples are adapted from the classical Denjoy example [5] (also see [10]) of a non-transitive $C^{1}$ diffeomorphism $h: S^{1} \rightarrow S^{1}$ of the circle with no periodic points. The property of the Denjoy map which we shall use is the following: there is a doubly infinite sequence $\left\{I_{k} \mid k=0, \pm 1, \pm 2, \ldots\right\}$ of pair $\because$ ise disjoint, non-degenerate, closed intervals such that $h\left(I_{k}\right)=I_{k+1}$ for all $k$ and $\bigcup_{-\infty}^{\infty} \partial I_{k} \subseteq \limsup _{k \rightarrow-\infty} I_{k}$; i.e. if $z \in \bigcup \partial I_{k}$, then every neighbourhood of $z$ meets infinitely many $I_{k}$ with $k<0$.

Actually, $\bigcup_{-\infty}^{\infty} I_{k}$ is dense and $\mathrm{cl}\left[\bigcup_{-\infty}^{\infty} \partial I_{k}\right]$ is a Cantor set which is the unique minimal set of $h$. However, we shall not make use of these facts.

Let $\pi:[0,1] \rightarrow S^{1}$ be a quotient map such that $\pi(0)=\pi(1)$ lies in $I_{0}$ and $\pi$ identifies no other pairs of points. Let $z \in I_{-1}$ be the unique point such that $h(z)=\pi(0)$. We can 'lift' $h$ to a (non-continuous) map $\tilde{h}:[0,1] \rightarrow[0,1]$ such that $\pi \circ \tilde{h}=h \circ \pi, \tilde{h}(0)=\tilde{h}(1)$ and $\tilde{h}$ has a single (jump) discontinuity at $\pi^{-1}(z)$ (figure 2).

Let $J_{k}=\pi^{-1}\left(I_{k}\right)$. Except for $J_{0}$, each $J_{k}$ is a non-degenerate, closed subinterval. The collection $\left\{J_{k} \mid k \geq 1\right\}$ is pairwise disjoint, $\tilde{h}\left(J_{k}\right)=J_{k+1}$ for all $k \geq 1$, and

$$
\bigcup_{-\infty}^{\infty} \partial J_{k} \subseteq \limsup _{k \rightarrow-\infty} J_{k} \text {. }
$$

(Here $\partial$ means 'boundary in $[0,1]$ ', so that $0,1 \notin \bigcup \partial J_{k}$.)

We now modify $\tilde{h}$ to eliminate the discontinuity at $\pi^{-1}(z)$, changing $\tilde{h}$ only in the interior of $J_{-1}$. Let $g:[0,1] \rightarrow[0,1]$ denote the resulting map (figure 3 ).

Since $\tilde{h}$ and $g$ disagree only in $J_{-1}^{\circ}$, it follows that whenever $x \notin \bigcup_{k \leq-1} J_{k}$, then $g^{n}(x)=\tilde{h}^{n}(x)$ for all $n \geq 0$. In particular, $g\left(J_{k}\right)=J_{k+1}$ for all $k \geq 1$.

We shall show in (4.2) that $g$ may be constructed so as to have homtervals.

We now define $f:[-1,1] \rightarrow[-1,1]$. Let $x_{0} \in(-1,0)$ and let $V=V_{L} \cup V_{R} \subseteq(-1,0)$ be a closed, connected neighbourhood of $x_{0}$. Define $f$ on $V$ so that:

(1) $f\left(x_{0}\right)$ is the left endpoint of $J_{1}$.

(2) $f\left(V_{R}\right) \subseteq J_{1}$.

(3) $f\left(V_{L}^{\circ}\right) \cap J_{1}=\varnothing$.

Define $f$ on $[0,1]$ by $f(x)=g(x)$ except for modifying $g$ on $J_{-1}^{\circ}$ so that:

(4) $V_{R} \subseteq f\left(J_{-1}\right) \subseteq\left[x_{0}, 1\right]$. 


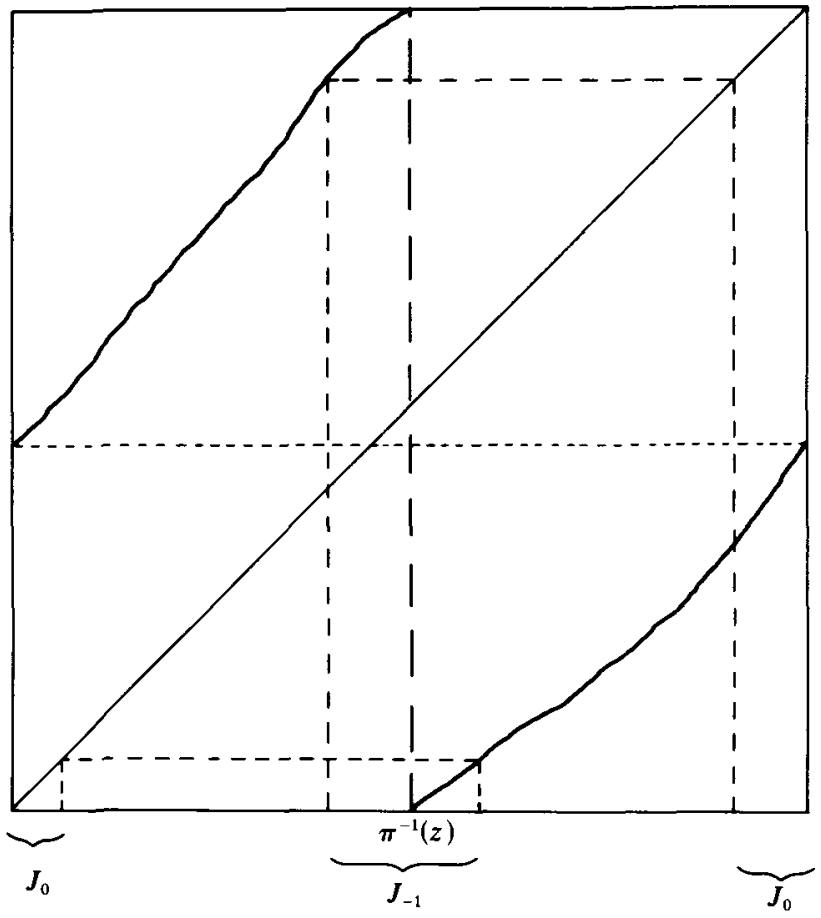

FIGURE 2

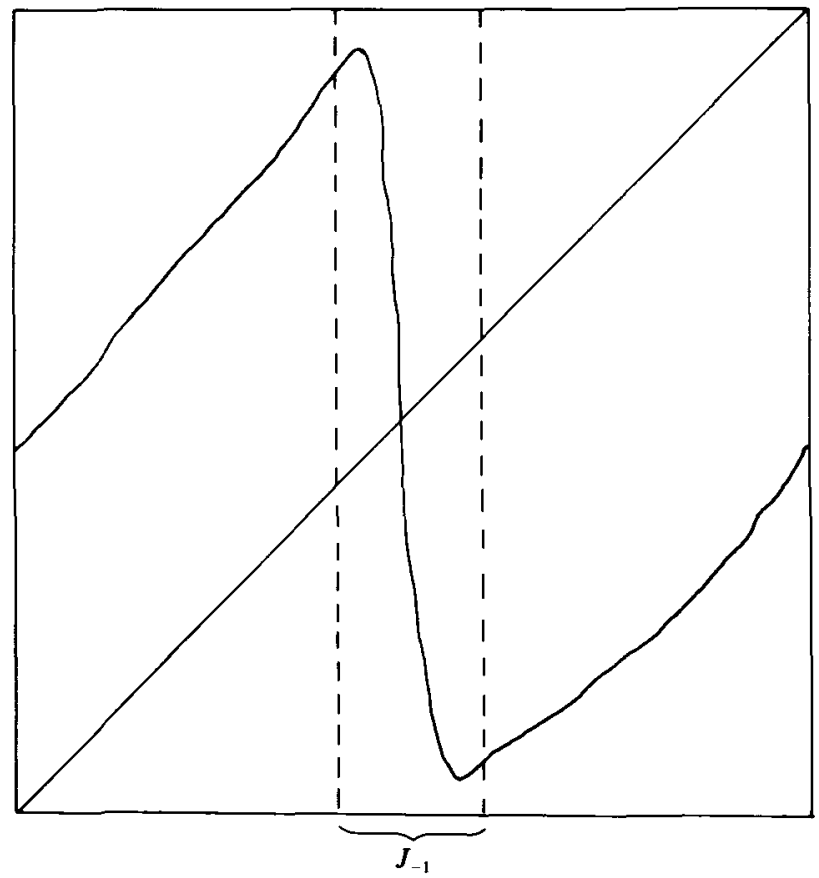

FIGURE 3 


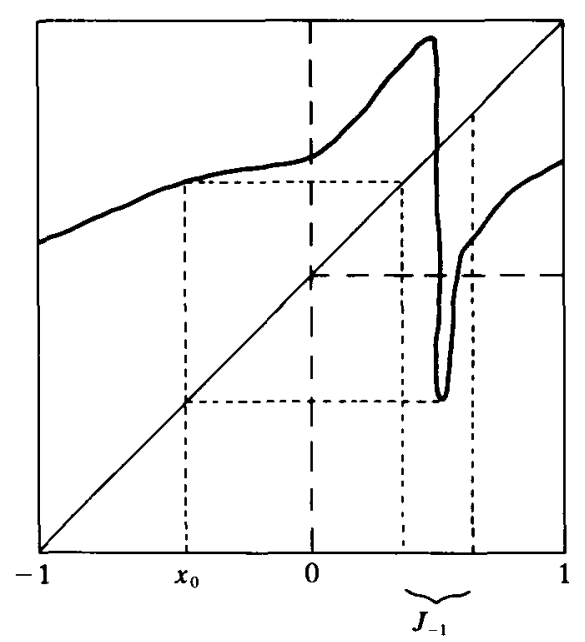

Figure 4

Finally, extend $f$ to all of $[-1,1]$ so that (figure 4 ):

(5) $f[-1,1] \cap V_{L}^{\circ}=\varnothing$.

Then

(6) $\left\{J_{k} \mid k \geq 1\right\}$ is a collection of pairwise disjoint, non-degenerate, closed subintervals such that $f\left(J_{k}\right)=J_{k+1}$ for all $k \geq 1$.

(7) $\bigcup_{-\infty}^{\infty} \partial J_{k} \subseteq \limsup _{k \rightarrow-\infty} J_{k}$.

(8) For every $n \geq 1, f^{n}\left(V_{R}\right) \subseteq f^{n-1}\left(J_{1}\right) \subseteq[0,1]$.

Let $W \subseteq V$ be a closed, connected neighbourhood of $x_{0}$. By (1) and (3), $f\left(W_{L}\right)$ contains an endpoint of $J_{1}$ as well as points not in $J_{1}$. Hence by (7), there exist arbitrarily large $n$ such that $J_{-n} \subseteq f\left(W_{L}\right)$. (If this were not the case, then the lengths of the intervals $J_{-n}$ would not approach zero.) Applying $f^{n-1}$ to this containment, we obtain:

(9) If $W \subseteq V$ is a closed, connected neighbourhood of $x_{0}$, then there exist arbitrarily large $n$ such that $J_{-1} \subseteq f^{n}\left(W_{L}\right)$.

(4.1) Proposition. With notation as above, $x_{0} \in \Omega-\bar{P}$ has an infinite orbit.

Proof. Since $f\left(x_{0}\right) \in J_{1}$, it follows from (6) that $x_{0}$ has an infinite orbit.

By (9) and (4), there are points arbitrarily close to $x_{0}$ whose orbit enters $J_{-1}$ and then returns arbitrarily close to $x_{0}$. Hence $x_{0} \in \Omega$.

By (8), any orbit which enters $V_{R}$ never returns to $V$, and by (9) and (4), any orbit which enters $V_{L}$ and then returns to $V$ returns in $V_{R}$. Thus no orbit enters $V$ more than twice. Hence $x \notin \bar{P}$.

We now return to the construction of $f$ and insert conditions which will ensure that $f$ is $C^{1}$ and has homtervals.

It is clear from the construction of $h$ (see [5] or [10]) that we may assume that: (10) $h^{\prime} \geq 1$ on $I_{k}$ if $k \leq 0$. 
We construct $\tilde{h}$ as before, noting that $\tilde{h}$ has no periodic points and that $\tilde{h}$ is $C^{1}$ everywhere except at the discontinuity. Thus when we modify $\tilde{h}$ to obtain $g$ we may require that $g$ be $C^{1}$ and that:

(11) If $x \in J_{-1}^{\circ}$ and $g(x) \notin J_{0}$, then $g^{\prime}(x) \leq-2$.

Then every $g$-periodic orbit is a source. For any such orbit enters $J_{-1}$ but not $J_{0}$. Therefore $\left|g^{\prime}\right| \geq 1$ everywhere on the orbit and $\left|g^{\prime}\right| \geq 2$ somewhere on the orbit.

Finally, construct $f$ as before, taking care that it be $C^{1}$.

(4.2) Proposition. The $C^{1}$ maps $f$ and $g$ have homtervals.

Proof. We show that $J_{1}$ is a homterval for $f$ and, a fortiori, for $g$.

If $J_{1}$ were contained in the domain of attraction of an $f$-periodic orbit, then by (8), this orbit would lie entirely in $[0,1]$. However, all $f$-periodic orbits contained in $[0,1]$ are $g$-periodic orbits as well, and all $g$-periodic orbits are sources. Thus $J_{1}$ cannot be contained in the demain of attraction of any periodic orbit for $f$ or $g$.

It now follows from (6) that $J_{1}$ is a homterval for $f$ and for $g$.

Since a $C^{2}$ diffeomorphism of the circle without periodic points must be transitive [5], the Denjoy example cannot be $C^{2}$. Thus our example cannot be $C^{2}$ either. It is unknown whether there are $C^{2}$ maps of the interval with homtervals.

\section{Unstable sets for fixed points}

We now turn our attention to non-wandering points with finite orbits. We shall see that any such point which is not itself periodic is homoclinic to a periodic point. By means of an analysis of the 'unstable sets' of the periodic point, we shall obtain information about the return times of any neighbourhood of the original point to itself. In this section, we concentrate on the properties of unstable sets which we shall find useful in the sequel.

Let $p$ be a fixed point of $f$. The unstable set of $p$ (under $f$ ) is

$$
\Lambda(p, f)=\bigcap_{V} \mathrm{cl}\left[\bigcup_{n \geq 0} f^{n}(V)\right]
$$

where the intersection is taken over all (basic) neighbourhoods of $p$. For $S=L$ or $R$, the one-sided unstable set of $p$ (under $f$ ) is

$$
\Lambda_{S}(p, f)=\bigcap_{V_{S}} \mathrm{cl}\left[\bigcup_{n \geq 0} f^{n}\left(V_{S}\right)\right]
$$

where the intersection is taken over all $S$-neighbourhoods of $p$.

We shall use abbreviated notation such as $\Lambda(p)$ or $\Lambda$ whenever there is no danger of confusion.

It is easy to see that $\Lambda(p)$ and $\Lambda_{S}(p)$ are closed (possibly degenerate) intervals containing $p$. Since $f(p)=p$, in either definition the union over $n \geq 0$ may be replaced by the union over $n \geq N$ for any pre-assigned $N$. It is clear that $\Lambda=\Lambda_{L} \cup \Lambda_{R}$.

We caution the reader that $\Lambda(p)$ need not be a neighbourhood (or even a one-sided neighbourhood) of $p$, and that $\Lambda_{L}$ need not be the 'left side' of $\Lambda$. In particular, any of 
the following kinds of behaviour may be observed: $\Lambda_{L}(p)$ is an $R$-neighbourhood of $p, \Lambda_{L}=\Lambda_{R}=\Lambda, \Lambda_{R}$ is a proper subset of $\Lambda_{L}$, etc.

In terms of sequences:

(5.1a) $x \in \Lambda(p, f)$ if and only if there exist $y_{i} \rightarrow p$ and $k_{i} \rightarrow \infty$ such that $f^{k_{i}}\left(y_{i}\right) \rightarrow x$.

(5.1b) $x \in \Lambda_{S}(p, f)$ if and only if there exist $y_{i} \stackrel{s}{\rightarrow} p$ and $k_{i} \rightarrow \infty$ such that $f^{k_{i}}\left(y_{i}\right) \rightarrow x$.

(Here $y_{i} \stackrel{L}{\rightarrow} p$ means that $y_{i} \rightarrow p$ and $y_{i} \leq p$ for sufficiently large $i ; y_{i} \stackrel{R}{\rightarrow} p$ is analogously defined.)

Using (5.1a) we see that $\Lambda$ is the (first) prolongation set (or equivalently in this case, prolongation limit set) of $p$, as defined by T. Ura [14] (also see [1] [2]).

Using the notation $\Lambda^{\circ}(p, f)$ for the interior of $\Lambda(p, f)$, we have:

(5.2a) $\Lambda^{\circ}(p, f) \subseteq \bigcap_{V} \bigcup_{n \geq 0} f^{n}(V)$.

(5.2b) $\Lambda_{s}^{\circ}(p, f) \subseteq \bigcap_{V_{s}} \bigcup_{n \geq 0} f^{n}\left(V_{s}\right)$.

(The sets on the right side of (5.2) are the unstable manifolds used by L. Block [3] in his work on maps of the interval.)

(5.3) Except possibly for $x=p, x \in \Lambda^{\circ}(p, f)$ if and only if $x \in \Lambda_{L}^{\circ}(p, f) \cup \Lambda_{R}^{\circ}(p, f)$.

(5.4) Lemma. $f\left[\Lambda_{S}(p, f)\right]=\Lambda_{S}(p, f)$ and hence $f[\Lambda(p, f)]=\Lambda(p, f)$.

Proof. If $y_{i} \stackrel{s}{\rightarrow} p, k_{i} \rightarrow \infty$ and $f^{k_{i}}\left(y_{i}\right) \rightarrow x$, then $f^{k_{i}+1}\left(y_{i}\right) \rightarrow f(x)$. Thus $f\left[\Lambda_{S}(p)\right] \subseteq \Lambda_{S}(p)$.

On the other hand, let $y \in \Lambda_{S}(p)$. We find $x \in \Lambda_{S}(p)$ with $f(x)=y$. We may assume that $y \neq p$ (otherwise choose $x=p$ ). There exist $y_{i} \stackrel{s}{\rightarrow} p$ and $k_{i} \rightarrow \infty$ such that $f^{k_{i}}\left(y_{i}\right) \rightarrow y$. Let $\left\{i^{\prime}\right\}$ be a subsequence such that $f^{k_{i}-1}\left(y_{i}\right)$ converges, say to $x$. Then $x \in \Lambda_{S}(p)$ and $f(x)=y$.

The property described in $(5.5 a)$ below clearly holds when $p$ is a source. The more careful argument required in the general case also gives a one-sided version $(5.5 b)$ which will be crucial in the proof of theorem 3 .

(5.5) Lemma. (a) If $x \in \Lambda^{\circ}(p, f)$, then for every neighbourhood $V$ of $p$, there exists $N \geq 0$ such that $x \in f^{n}(V)$ for all $n \geq N$.

(b) If $x \in \Lambda_{s}^{\circ}(p, f)$, then for every $S$-neighbourhood $V_{s}$ of $p$, there exists $N \geq 0$ such that $x \in f^{2 n+N}\left(V_{S}\right)$ for all $n \geq 0$.

Proof. We shall show

(b) If $x \in \Lambda_{s}^{\circ}(p, f)$, then one of the following statements holds:

(i) For every closed, connected neighbourhood $V=V_{S} \cup V_{T}$ of $p$, there exists $N \geq 0$ such that $x \in f^{n}\left(V_{S}\right)$ for all $n \geq N$.

(ii) For every closed, connected neighbourhood $V=V_{S} \cup V_{T}$ of $p$, there exists $N \geq 0$ such that $x \in f^{2 n+N}\left(V_{S}\right)$ and $x \in f^{2 n+N+1}\left(V_{T}\right)$ for all $n \geq 0$.

Clearly $\left(b^{\prime}\right)$ implies $(b)$ and, by (5.3), $(a)$ as well.

To prove $\left(b^{\prime}\right)$, let $x \in \Lambda_{s}^{\circ}(p)$. We may assume that $x \neq p$, for otherwise there is nothing to prove. Let $V_{0}$ be a neighbourhood of $p$ such that $x \notin V_{0}$. We consider only neighbourhoods $V$ of $p$ small enough so that $V \cup f(V) \subseteq V_{0}$. 
Let $V=V_{S} \cup V_{T}$ be such a closed, connected neighbourhood of $p$. Then $f\left(V_{s}\right) \nsubseteq V_{S}$, for otherwise $\bigcup f^{n}\left(V_{S}\right) \subseteq V_{0}$ and hence by $(5.2 b), x \in V_{0}$. Therefore one of the following statements holds:

(1) $f\left(V_{s}\right) \supseteq V_{s}$.

(2) $f\left(V_{S}\right)=W_{S} \cup W_{T}$ where $W_{S} \subseteq V_{S}$ and $W_{T}$ is non-degenerate.

If there are arbitrarily small $V$ for which (1) holds, then (i) holds for all $V$. Suppose then that (2) holds for all sufficiently small $V$. Since $V_{S} \cup W_{T} \subseteq V \cup f(V) \subseteq V_{0}$, then as above, $f\left(V_{S} \cup W_{T}\right) \notin V_{S} \cup W_{T}$ and, in particular, $f\left(W_{T}\right) \notin V_{S} \cup W_{T}$. Therefore one of the following statements holds:

(3) $f\left(W_{T}\right) \supseteq W_{T}$.

(4) $f\left(W_{T}\right) \supseteq V_{S}$.

Suppose there are arbitrarily small $V$ for which (3) holds. For any such $V$, since $x \notin V_{S}$ and $x \in f^{N}\left(V_{S}\right)$ for some $N \geq 1$, it follows from (2) that $x \in f^{n}\left(W_{T}\right)$ for all $n \geq N-1$, and hence that $x \in f^{n}\left(V_{S}\right)$ for all $n \geq N$. Thus there are arbitrarily small $V$ for which (i) holds. Hence (i) holds for all $V$.

Suppose then that (2) and (4) hold for all sufficiently small $V$. For any such $V$, $f^{2}\left(V_{s}\right) \supseteq V_{s}$. Since $x \in f^{N}\left(V_{s}\right)$ for some $N \geq 1$, it follows that (ii) holds for all sufficiently small $V$ and hence for all $V$.

\section{Light maps}

We will prove theorem 3 by induction on the period of the periodic point in the orbit of $x$. The first step is to show that if $x \in \Omega$ and the orbit of $x$ contains a fixed point of $f$, then $x \in \Omega\left(f^{n}\right)$ for all odd $n \geq 1$. The argument is simpler and the result stronger ('all odd' becomes 'all') when $f$ is a 'light' map. We devote this section to a digression on such maps. The results of this section will not be used elsewhere in the paper.

Recall that a map is light if inverse images of points are totally disconnected. For maps of the interval, $f$ is light if for every non-degenerate subinterval $J \subseteq I, f(J)$ is non-degenerate; equivalently, $f$ is light if for every $x \in I$ and every neighbourhood $U$ of $x, f(U)$ contains a one-sided neighbourhood of $f(x)$.

We call $f$ open at $x$ if for every neighbourhood $U$ of $x, f(U)$ is a neighbourhood of $f(x)$.

The following result was proved in [11] for piecewise monotone maps, which are open at all points except (possibly) critical points and endpoints.

(6.1) LEMMA. If $f$ is light and $x \in \Omega-\bar{P}$, then every power of $f$ is open at $x$.

Proof. If $f^{n}$ is not open at $x$ for some $n \geq 1$, then there is a closed, connected neighbourhood $U$ of $x$, disjoint from $P$, such that either $f^{n}(U)$ is degenerate or $f^{n}(x)$ is an endpoint of $f^{n}(U)$. Since $f$, and hence $f^{n}$, is light, the first case cannot occur.

In the second case, $f^{n}(U)$ is a one-sided neighbourhood of $f^{n}(x)$, and in fact $f^{n}$ maps both sides of $U=U_{L} \cup U_{R}$ to the same side of $f^{n}(x)$. Since $x \in \Omega-\bar{P}$, by (2.7), $x$ is not an endpoint of $I$ and hence neither $U_{L}$ nor $U_{R}$ is degenerate. By shrinking $U$, we may assume that $f^{n}(U)=f^{n}\left(U_{L}\right)=f^{n}\left(U_{R}\right)$.

By (2.6), there exist $y \in U$ and $m \geq n$ such that $f^{m}(y)=x$. Without loss of generality, $y \in U_{L}$. Then there exists $z \in U_{R}$ such that $f^{n}(y)=f^{n}(z)$, and hence 
$f^{m}(z)=x$ as well. But then $f^{m}(y)<y$ and $f^{m}(z)>z$. By (2.4), $U \cap P \neq \varnothing$, a contradiction.

(6.2) LEMMA. For light maps, if $x \in \Omega$ and the orbit of $x$ contains a fixed point of $f$, then $x \in \Omega\left(f^{n}\right)$ for all $n \geq 1$.

Proof. If $x \in \bar{P}$, there is nothing to prove. So we assume that $x \in \Omega-\bar{P}$.

Let $p=f^{m}(x)$ be the (unique) fixed point in the orbit of $x$. Since $x \in \Omega$, there exist $x_{i} \rightarrow x$ and $k_{i} \rightarrow \infty$ such that $f^{k_{i}}\left(x_{i}\right) \rightarrow x$. Then $y_{i}=f^{m}\left(x_{i}\right) \rightarrow p$ and $f^{k_{i}-m}\left(y_{i}\right) \rightarrow x$. Therefore $x \in \Lambda(p)$.

Now let $U$ be a neighbourhood of $x$. It suffices to show that $f^{i}(U) \cap U \neq \varnothing$ for all sufficiently large $j$. Since $x \neq p$ and $\Lambda(p)$ is an interval containing both $x$ and $p$, it follows that $U \cap \Lambda^{\circ}(p) \neq \varnothing$. Let $z \in U \cap \Lambda^{\circ}(p)$. By (6.1), $f^{m}(U)$ is a neighbourhood of $p$, hence by $(5.5 a)$ applied to $z$ and $f^{m}(U)$, there exists $N \geq 1$ such that $z \in f^{i}\left[f^{m}(U)\right]$ for all $j \geq N$. Hence $f^{j}(U) \cap U \neq \varnothing$ for all $j \geq N+m$.

As an easy consequence of (6.2), we have the following.

THEOREM 2. For light maps, if $x \in \Omega\left(f^{N}\right)$ and the f-orbit of $x$ contains a fixed point of $f^{N}$, then $x \in \Omega\left(f^{n}\right)$ for all $n \geq 1$.

Proof. Since $f$ is light, so is $f^{N}$. Hence by (6.2), $x \in \Omega\left(f^{n N}\right)$ for all $n \geq 1$. Therefore $x \in \Omega\left(f^{n}\right)$ for all $n \geq 1$.

\section{Another example}

The example of $\S 1$ is typical of light maps with $\Omega(f) \neq \Omega\left(f^{n}\right)$ for some $n \geq 2$. The following example shows that more complicated behaviour may occur for non-light maps (figure 5).

Let $f:[0,1] \rightarrow[0,1]$ be differentiable and let $0<x_{L}<x_{R}<p<c<1$ satisfy:

(1) $f\left(x_{L}\right)=p, f\left(x_{R}\right)=p, f(p)=p, f(c)=x_{L}$.

(2) $f$ is increasing in some $L$-neighbourhood of $x_{L}$ and some $R$-neighbourhood of $x_{R}, f^{\prime} \equiv 0$ on $\left(x_{L}, x_{R}\right), f^{\prime}(p)<-1, f^{\prime}(c)=0$.

(3) $f\left[0, x_{L}\right] \subseteq\left[x_{R}, p\right], \quad f\left[x_{L}, x_{R}\right]=p, \quad f\left[x_{R}, p\right] \subseteq[p, 1], \quad f[p, c]=\left[x_{L}, p\right], \quad f[c, 1] \subseteq$ $\left[x_{L}, x_{R}\right]$.

(4) For every $L$-neighbourhood $V_{L}$ of $p, c \in f^{2 n+1}\left(V_{L}\right)$ for some $n \geq 0$.

(7.1) Proposition. With notation as above, $x_{L} \in \Omega(f)-\Omega\left(f^{2}\right)$.

Proof. Let $U$ be a closed, connected neighbourhood of $x_{L}$ small enough so that $f$ is increasing on $U_{L}$ and $U_{R} \subseteq\left[x_{L}, x_{R}\right]$. Then $f(U)$ is an $L$-neighbourhood of $p$ and hence $f^{2}(U) \subseteq f\left[x_{R}, p\right] \subseteq[p, 1]$. Since $f^{2}[p, 1] \subseteq[p, 1]$, it follows that $x_{L} \notin \Omega\left(f^{2}\right)$.

On the other hand, by $(4) c \in f^{2 n}(U)$ for some $n \geq 1$. Hence $x_{L} \in f^{2 n+1}(U)$ and so $x_{L} \in \Omega(f)$.

It is easy to see why (6.2) fails for this example: $f$ is not open at $x_{L}$ (the image of a small neighbourhood of $x_{L}$ is only an $L$-neighbourhood of $p$ ) and so we cannot apply (5.5a) to conclude that $x \in \Omega\left(f^{n}\right)$ for all $n \geq 1$. However, we could apply $(5.5 b)$ and discover that $x \in \Omega\left(f^{n}\right)$ for all odd $n \geq 1$. Our proof of theorem 3 will reduce to such 


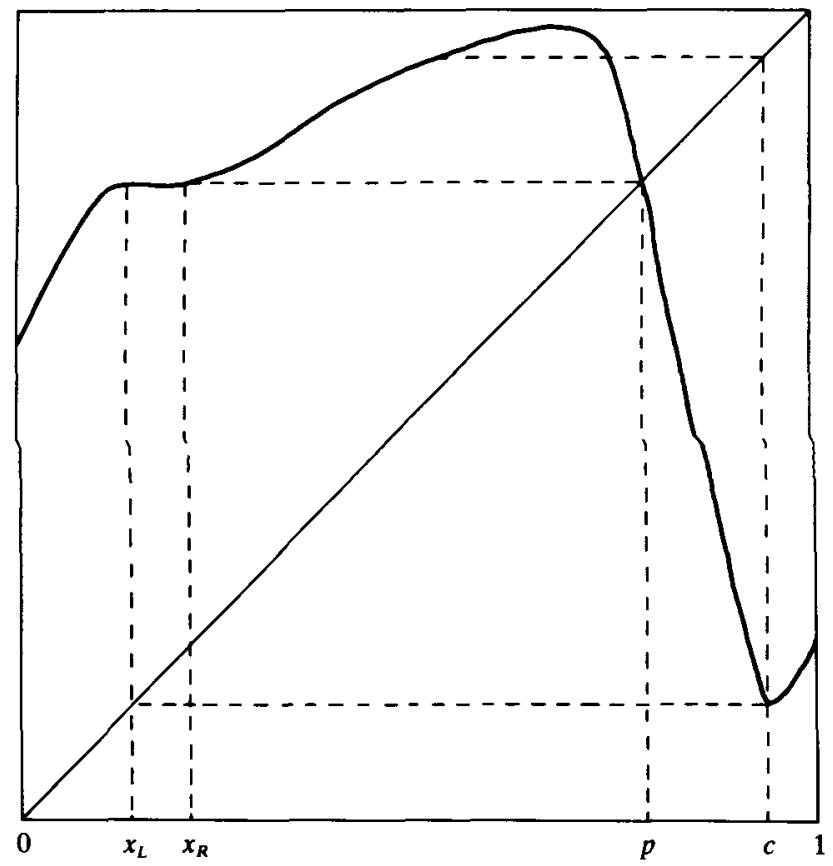

Figure 5

an argument. The reduction is based on a more detailed study of the unstable sets $\Lambda_{S}\left(p, f^{N}\right)$ of fixed points of $f^{N}$.

\section{Unstable sets for periodic points}

In this section, we shall prove some technical results about the relations among the various unstable sets $\Lambda_{S}\left(p, f^{N}\right)$ as $p$ ranges over the $f$-orbit of a fixed point of $f^{N}$. These results will be used in the proof of theorem 3 in the next section. For notational convenience, in the proofs we will abbreviate $\Lambda_{S}\left(p, f^{N}\right)$ to $\Lambda_{S}(p)$.

Throughout this section, $p$ and $q$ will denote fixed points of $f^{N}$.

(8.1) LEMMA. Let $q=f^{r}(p)$. If for every $S$-neighbourhood $V_{S}$ of $p, f^{r}\left(V_{S}\right)$ contains a $T$-neighbourhood of $q$, then $f^{r}\left[\Lambda_{S}\left(p, f^{N}\right)\right] \supseteq \Lambda_{T}\left(q, f^{N}\right)$. If, furthermore, for every sufficiently small such $V_{S}, f^{r}\left(V_{S}\right)$ is a T-neighbourhood of $q$, then $f^{r}\left[\Lambda_{S}\left(p, f^{N}\right)\right]=$ $\Lambda_{T}\left(q, f^{N}\right)$.

Proof. To prove the first statement, let $y \in \Lambda_{T}(q)$. Without loss of generality, $y \neq p$. There exists $y_{i} \stackrel{r}{\rightarrow} q$ and $k_{i} \rightarrow \infty$ such that $f^{k_{i} N}\left(y_{i}\right) \rightarrow y$. Choose $x_{i} \stackrel{s}{\rightarrow} p$ with $f^{r}\left(x_{i}\right)=y_{i}$. By passing to a subsequence, we may assume that $f^{k_{i} N}\left(x_{i}\right)$ converges, say to $x$. Then $x \in \Lambda_{S}(p)$ and $f^{r}(x)=y$.

To prove the second statement, let $x \in \Lambda_{S}(p)$. There exist $x_{i} \stackrel{s}{\rightarrow} p$ and $k_{i} \rightarrow \infty$ such that $f^{k_{i} N}\left(x_{i}\right) \rightarrow x$. Then $f^{r}\left(x_{i}\right) \stackrel{T}{\rightarrow} q$ and $f^{k_{i} N}\left[f^{r}\left(x_{i}\right)\right] \rightarrow f^{r}(x)$. Therefore $f^{r}(x) \in \Lambda_{T}(q)$. 
We remark that for piecewise monotone maps, if the first condition holds, then so does the second.

(8.2) LEMMA. Let $q=f^{r}(p)$. If $\Lambda_{S}\left(p, f^{N}\right)$ is non-degenerate, then there exists a side $T$ such that

(i) For every $S$-neighbourhood $V_{S}$ of $p, f^{r}\left(V_{S}\right)$ contains a $T$-neighbourhood of $q$.

(ii) $f^{\prime}\left[\Lambda_{S}\left(p, f^{N}\right)\right]=\Lambda_{T}\left(q, f^{N}\right)$ is non-degenerate.

Proof. At least one side $T$ satisfying (i) must exist, for otherwise $\Lambda_{S}(p)$ would be degenerate. Furthermore, it follows from (5.4) that $f^{r}\left[\Lambda_{S}(p)\right]$ is non-degenerate. Thus it suffices to show that $f^{r}\left[\Lambda_{S}(p)\right]=\Lambda_{T}(q)$ for some $T$ satisfying (i).

If for every sufficiently small $S$-neighbourhood $V_{S}$ of $p, f^{r}\left(V_{S}\right)$ is a $T$-neighbourhood of $q$, then by $(8.1), f^{r}\left[\Lambda_{S}(p)\right]=\Lambda_{T}(q)$.

We therefore suppose that for every $S$-neighbourhood $V_{S}$ of $p, f^{\prime}\left(V_{S}\right)$ is a neighbourhood of $q$. Then both $T=L$ and $T=R$ satisfy (i). Furthermore, by applying the argument in the proof of the second statement of $(8.1)$ to $\Lambda(q)$ rather than $\Lambda_{T}(q)$, we may conclude that $f^{r}\left[\Lambda_{S}(p)\right]=\Lambda(q)$. Therefore

(1) $f^{\prime}\left[\Lambda_{S}(p)\right]=\Lambda_{L}(q) \cup \Lambda_{R}(q)$.

Choose $m \geq 0$ so that $r+m$ is a multiple of $N$. Then at least one of the following statements is true.

(2) For at least one choice of $T, f^{m}\left[\Lambda_{T}(q)\right]$ contains an $S$-neighbourhood of $p$.

(3) For both choices of $T, f^{m}\left[\Lambda_{T}(q)\right]$ contains no $S$-neighbourhood of $p$.

If (2) holds, then $f^{m}\left[\Lambda_{T}(q)\right] \supseteq \Lambda_{S}(p)$. Applying $f^{r}$ to this containment and using (5.4), we get $\Lambda_{T}(q) \supseteq f^{r}\left[\Lambda_{S}(p)\right]$. The equality in (ii) then follows from (1).

If (3) holds, then the intervals $f^{m}\left[\Lambda_{L}(q)\right]$ and $f^{m}\left[\Lambda_{R}(q)\right]$ have $p$ as their common $S$-endpoint. Therefore, one is contained in the other. Let $T$ correspond to the larger set. Again the equality in (ii) follows from (1).

(8.3) LEMMA. If $f^{r}(p) \in \Lambda_{S}^{\circ}\left(p, f^{N}\right)$, then $f^{r}\left[\Lambda_{S}\left(p, f^{N}\right)\right]=\Lambda_{S}\left(p, f^{N}\right)$.

Proof. It suffices to show that $f^{r}\left[\Lambda_{S}(p)\right] \subseteq \Lambda_{S}(p)$. For then, applying $f^{r}$ to this containment $N-1$ times, we obtain

$$
f^{r N}\left[\Lambda_{S}(p)\right] \subseteq \cdots \subseteq f^{r}\left[\Lambda_{S}(p)\right] \subseteq \Lambda_{S}(p)
$$

Since the ends are equal by $(5.4), f^{r}\left[\Lambda_{S}(p)\right]=\Lambda_{S}(p)$.

So, let $x \in f^{r}\left[\Lambda_{S}(p)\right]=\Lambda_{T}(q)$, where $q=f^{r}(p)$ and $T$ is given by (8.2). Let $U$ be a neighbourhood of $x$ and let $V_{S}$ be an $S$-neighbourhood of $p$. Since $q \in \Lambda_{S}^{\circ}(p)$, there exists $k \geq 0$ such that $f^{k N}\left(V_{S}\right)$ contains a neighbourhood of $q$ and, in particular, contains a $T$-neighbourhood $W_{T}$ of $q$. Since $x \in \Lambda_{T}(q)$, there exists $j \geq 0$ such that $U \cap f^{j N}\left(W_{T}\right) \neq \varnothing$. Then $U \cap f^{(j+k) N}\left(V_{S}\right) \neq \varnothing$ and hence $x \in \Lambda_{S}(p)$.

(8.4) LEMMA. Let $1 \leq r \leq N-1$. If $q=f^{r}(p) \in \Lambda_{S}\left(p, f^{N}\right)$, then either $f^{2 r}(p)=p$ or $\Lambda_{S}\left(p, f^{N}\right)=\Lambda_{T}\left(q, f^{N}\right)$ where $T$ is as in (8.2).

Proof. We first prove (8.4) assuming that $q$ is the left-most point of the $f$-orbit of $p$.

We may assume that $p, q, f^{r}(q)$ are distinct, for otherwise $f^{2 r}(p)=p$. Then one of the following statements holds:

(4) $q<p<f^{r}(q)$.

(5) $q<f^{r}(q)<p$. 
Suppose (4) holds. Since $f^{r}(q) \in f^{r}\left[\Lambda_{S}(p)\right]=\Lambda_{T}(q)$ and $q \in \Lambda_{T}(q)$, it follows that $p \in \Lambda_{T}^{\circ}(q)$. Since $p=f^{N-r}(q)$, it follows from (8.3) that $f^{N-r}\left[\Lambda_{T}(q)\right]=\Lambda_{T}(q)$. By (8.2), $f^{N-r}\left[\Lambda_{T}(q)\right]=\Lambda_{S}(p)$. Therefore $\Lambda_{S}(p)=\Lambda_{T}(q)$.

Suppose (5) holds. Then $f^{2 r}(p)=f^{r}(q) \in \Lambda_{S}^{\circ}(p)$. Then by $(8.3), f^{2 r}\left[\Lambda_{S}(p)\right]=\Lambda_{S}(p)$. By (8.2), $f^{2 r}\left[\Lambda_{S}(p)\right]=f^{r}\left[\Lambda_{T}(q)\right]$. Therefore $\Lambda_{S}(p)=f^{r}\left[\Lambda_{T}(q)\right]$, and hence $p=$ $f^{N-r}(q) \in f^{N-r}\left[\Lambda_{S}(p)\right]=\Lambda_{T}(q)$. Thus $f^{r}(q) \in \Lambda_{T}^{\circ}(q)$. By $(8.3), f^{r}\left[\Lambda_{T}(q)\right]=\Lambda_{T}(q)$ and hence $\Lambda_{S}(p)=\Lambda_{T}(q)$.

This completes the proof when $q$ is the left-most point of the $f$-orbit of $p$.

To prove (8.4) in general, let $q^{\prime}=f^{r}\left(p^{\prime}\right) \in \Lambda_{S^{\prime}}\left(p^{\prime}\right)$ where $p^{\prime}$ is an arbitrary point of the $f$-orbit of $p$. We show that either $f^{2 r}\left(p^{\prime}\right)=p^{\prime}$ or $\Lambda_{S^{\prime}}\left(p^{\prime}\right)=\Lambda_{T^{\prime}}\left(q^{\prime}\right)$ where $T^{\prime}$ is given by (8.2) using $p^{\prime}$ in place of $p$ and $S^{\prime}$ in place of $S$.

There exists $r^{\prime}, 0 \leq r^{\prime} \leq N-1$, such that $p=f^{r^{\prime}}\left(p^{\prime}\right)$ and $q=f^{r^{\prime}}\left(q^{\prime}\right)$. We may assume that $r^{\prime} \neq 0$, for otherwise $p=p^{\prime}$ and there is nothing more to prove. Let $S^{\prime \prime}$ be given by (8.2) using $p^{\prime}$ in place of $p, r^{\prime}$ in place of $r$, and $S^{\prime}$ in place of $S$. Then

(6) $\Lambda_{S^{\prime \prime}}(p)=f^{r^{\prime}}\left[\Lambda_{S^{\prime}}\left(p^{\prime}\right)\right]$.

Since $q \in \Lambda_{S^{\prime \prime}}(p)$, either $f^{2 r}(p)=p$, in which case $f^{2 r}\left(p^{\prime}\right)=p^{\prime}$, or $\Lambda_{S^{\prime \prime}}(p)=\Lambda_{T^{\prime \prime}}(q)$ where $T^{\prime \prime}$ is given by (8.2) using $S^{\prime \prime}$ in place of $S$. Then

(7) $\Lambda_{T^{\prime \prime}}(q)=f^{r^{\prime}}\left[\Lambda_{T^{\prime}}\left(q^{\prime}\right)\right]$.

Since $f^{N-r^{\prime}}\left[\Lambda_{\mathcal{S}^{\prime \prime}}(p)\right]=f^{N-r^{\prime}}\left[\Lambda_{T^{\prime \prime}}(q)\right]$, it follows from $(6)$ and $(7)$ that $\Lambda_{S^{\prime}}\left(p^{\prime}\right)=$ $\Lambda_{T^{\prime}}\left(q^{\prime}\right)$.

\section{Theorem 3}

In this section, we prove

THEOREM 3. If $x \in \Omega$ has a finite orbit, then $x \in \Omega\left(f^{n}\right)$ for all odd $n \geq 1$.

The proof of theorem 3 is by induction (on $N$ ) in the following statement:

If $x \in \Omega$ and the $f$-orbit of $x$ contains a fixed point of $f^{N}$, then $x \in \Omega\left(f^{n}\right)$ for all odd $n \geq 1$.

For $N=1,2$, (indeed for any power of 2$),(*)$ is a consequence of the following result.

(9.1) LEMMA. If $x \in \Omega$ and the orbit of $x$ contains a fixed point of $f^{N}$, then $x \in \Omega\left(f^{n}\right)$ for all $n$ relatively prime to $2 N$.

Proof. We may suppose that $x \in \Omega-\bar{P}$, for if $x \in \bar{P}$, then $x \in \Omega\left(f^{n}\right)$ for all $n \geq 1$.

As in the proof of (6.2), let $q=f^{i}(x)$ be a fixed point of $f^{N}$ in the $f$-orbit of $x$. Since $x \in \Omega$, there exist $x_{i} \rightarrow x$ and $k_{i} \rightarrow \infty$ such that $f^{k_{i}}\left(x_{i}\right) \rightarrow x$. Then $y_{i}=f^{j}\left(x_{i}\right) \rightarrow q$ and $f^{k_{i}-i}\left(y_{i}\right) \rightarrow x$. By choosing a subsequence, we may assume that all the $k_{i}$ lie in the same residue class modulo $N$, say $k_{i} \equiv k \geq j$. Then $z_{i}=f^{k}\left(x_{i}\right) \rightarrow f^{k}(x)=p$, and $f^{k_{i}-k}\left(z_{i}\right) \rightarrow x$. By choosing a further subsequence, we can find a side $S$ such that $z_{i} \stackrel{s}{\rightarrow} p$. Hence $x \in \Lambda_{S}\left(p, f^{N}\right)$.

Summarizing, we have found $p, k$ and $S$ such that:

(1) $f^{k}(x)=p$.

(2) $f^{N}(p)=p$.

(3) $x \in \Lambda_{S}\left(p, f^{N}\right)$. 
(4) For every neighbourhood $U$ of $x, f^{k}(U)$ contains an $S$-neighbourhood of $p$.

It is crucial here that $S$ be the same in (3) and (4).

Now let $U$ be a neighbourhood of $x$. Since $\Lambda_{S}\left(p, f^{N}\right)$ is an interval, which by (3) contains $x, U \cap \Lambda_{s}^{\circ}\left(p, f^{N}\right) \neq \varnothing$. Let $z$ be any point in this intersection. By $(4), f^{k}(U)$ contains an $S$-neighbourhood of $p$. Then by $(5.5 b)$ applied to $z \in \Lambda_{S}^{\circ}\left(p, f^{N}\right)$ and $f^{k}(U)$, there exists $M \geq 0$ such that $z \in f^{(2 m+M) N}\left[f^{k}(U)\right]$ for all $m \geq 0$. Therefore $U \cap f^{2 m N+(k+M N)}(U) \neq \varnothing$ for all $m \geq 0$. Since $\{2 m N+(k+M N) \mid m \geq 0\}$ contains multiples of all $n$ relatively prime to $2 N$, it follows that $x \in \Omega\left(f^{n}\right)$ for all such $n$.

To complete the proof of theorem 3 , suppose that $(*)$ is true for all maps of the interval and all $M \leq N-1$ where $N \geq 3$. We prove (*) holds for $N$.

Let $x \in \Omega(f)$ and suppose that the $f$-orbit of $x$ contains a fixed point of $f^{N}$. We may assume that $N$ is the least $f$-period of this point. As in the proof of (9.1), there is a point $p$, an integer $k \geq 1$, and a side $S$ such that:

(5) $f^{k}(x)=p$.

(6) $f^{N}(p)=p$.

(7) $x \in \Lambda_{s}\left(p, f^{N}\right)$.

(8) For every neighbourhood $U$ of $x, f^{k}(U)$ contains an $S$-neighbourhood of $p$.

Suppose that $k$ and $N$ have a common factor $m \geq 2$. Let $k=m l, N=m M$ and $g=f^{m}$. Then

(5') $g^{l}(x)=p$.

(6') $g^{M}(p)=p$.

(7) $x \in \Lambda_{s}\left(p, g^{M}\right)$.

(8') For every neighbourhood $U$ of $x, g^{l}(U)$ contains an $S$-neighbourhood of $p$.

It follows from $\left(7^{\prime}\right)$ and $\left(8^{\prime}\right)$ that $x \in \Omega(g)$, and from $\left(5^{\prime}\right)$ and $\left(6^{\prime}\right)$ that the $g$-orbit of $x$ contains a fixed point of $g^{M}$. But $M \leq N-1$, so by the inductive hypothesis, $x \in \Omega\left(g^{n}\right)$ for all odd $n \geq 1$. Therefore $x \in \Omega\left(f^{n}\right)$ for all odd $n \geq 1$.

Suppose then that $k$ and $N$ are relatively prime. Let $j N$ be the least multiple of $N$ greater than $k$. Then $j N=k+r$ where $1 \leq r \leq N-1$, and $r$ and $N$ are relatively prime. Let $q=f^{j N}(x)$. Then

(9) $q \in f^{j N}\left[\Lambda_{S}\left(p, f^{N}\right)\right]=\Lambda_{S}\left(p, f^{N}\right)$.

Since $q=f^{N-r}(p)$, by (8.2) there exists a side $T$ such that:

(10) For every $S$-neighbourhood $V_{S}$ of $p, f^{N-r}\left(V_{S}\right)$ contains a $T$-neighbourhood of $q$.

(11) $f^{N-r}\left[\Lambda_{S}\left(p, f^{N}\right)\right]=\Lambda_{T}\left(q, f^{N}\right)$.

Then by (8.4), either $f^{2 r}(p)=p$ or $\Lambda_{S}(p)=\Lambda_{T}(q)$. If $f^{2 r}(p)=p$, then, since $N$ is the least $f$-period of $p, N=2 r$. But $r$ and $N$ are relatively prime, so $N=2$. However, we have assumed that $N \geq 3$. Therefore $\Lambda_{S}(p)=\Lambda_{T}(q)$ and

$\left(5^{\prime \prime}\right) f^{j N}(x)=q$.

$\left(6^{\prime \prime}\right) f^{N}(q)=q$.

(7") $x \in \Lambda_{T}\left(q, f^{N}\right)$.

$\left(8^{\prime \prime}\right)$ For every neighbourhood $U$ of $x, f^{i N}(U)$ contains a $T$-neighbourhood of $q$.

Thus $x \in \Omega\left(f^{N}\right)$ and the $f^{N}$-orbit of $x$ contains a fixed point of $f^{N}$. Then by (9.1), $x \in \Omega\left(f^{n N}\right)$ for all odd $n \geq 1$ and hence $x \in \Omega\left(f^{n}\right)$ for all odd $n \geq 1$. 
This completes the proof of theorem 3 and hence of the main theorem.

10. The sets $\Omega\left(f^{n}\right)$

It follows from the main theorem that if $n=2^{k} m$ where $m$ is odd, then $\Omega\left(f^{n}\right)=$ $\Omega\left(f^{2 k}\right)$. Thus all the possible sets $\Omega\left(f^{n}\right)$ appear in the nested sequence

$$
\Omega(f) \supseteq \Omega\left(f^{2}\right) \supseteq \Omega\left(f^{4}\right) \supseteq \cdots
$$

In this section, we shall show how to realize any pre-assigned sequence of equalities and strict containments in (*).

Our main tool is the 'return time doubling' construction of P. Štefan [13]. Starting with a map $f: I \rightarrow I$, it produces a map $g: J \rightarrow J$ where $J \supseteq I$ and $g^{2} \mid I=f$. We illustrate this construction with $I=[0,1]$ and $J=[0,3]$. Define $g$ as follows:

$$
g(x)= \begin{cases}f(x)+2 & \text { if } x \in[0,1] \\ x-2 & \text { if } x \in[2,3]\end{cases}
$$

and linearly on $[1,2]$ (figure 6).
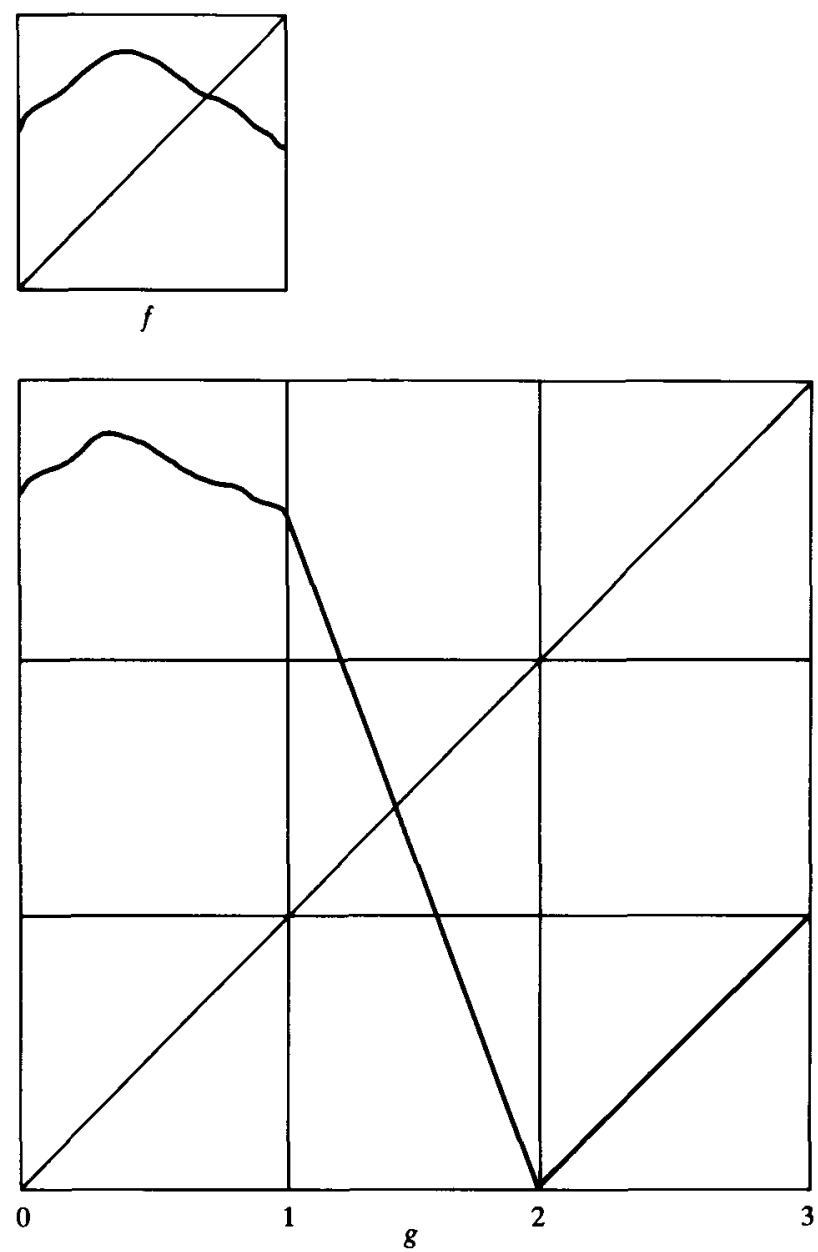

FIGURE 6 
Then $\Omega(g)=\Omega(f) \cup\{x+2 \mid x \in \Omega(f)\} \cup\{p\}$ where $p$ is the unique fixed point of $g$ in $(1,2)$. Furthermore, the return times under $g$ of the non-wandering points in $[0,1]$ are twice the return times under $f$.

Now consider the map $f=f_{0}$ of $\S 1$. By theorem 2:

(1) $\Omega\left(f_{0}\right) \supsetneqq \Omega\left(f_{0}^{2}\right)=\Omega\left(f_{0}^{4}\right)=\cdots$

Applying Štefan's construction to $f_{0}$, we obtain a map $f_{1}$ satisfying:

(2) $\Omega\left(f_{1}\right)=\Omega\left(f_{1}^{2}\right) \supsetneq \Omega\left(f_{1}^{4}\right)=\Omega\left(f_{1}^{8}\right)=\cdots$

Iterating this construction $n$ times, we obtain a map $f_{n}$ with exactly one strict containment in the sequence $(*)$ :

(3) $\Omega\left(f_{n}\right)=\cdots=\Omega\left(f_{n}^{2 n}\right) \supsetneqq \Omega\left(f_{n}^{2^{n+1}}\right)=\cdots$

It is now easy to construct a map $f$ with any finite set of strict containments in $(*)$. Suppose that the strict containments are to follow the sets $\Omega\left(f^{2^{n}}\right)$ for $n=n_{1}, \ldots, n_{m}$. Let $I_{1}, \ldots, I_{m}$ be pairwise disjoint, closed intervals (for convenience, let $I_{k+1}$ lie to the right of $I_{k}$ ) and define $f$ so that $f \mid I_{k}$ is conjugate to the map $f_{n_{k}}$ defined above. Then define $f$ linearly between the $I_{k}$ s (figure 7).

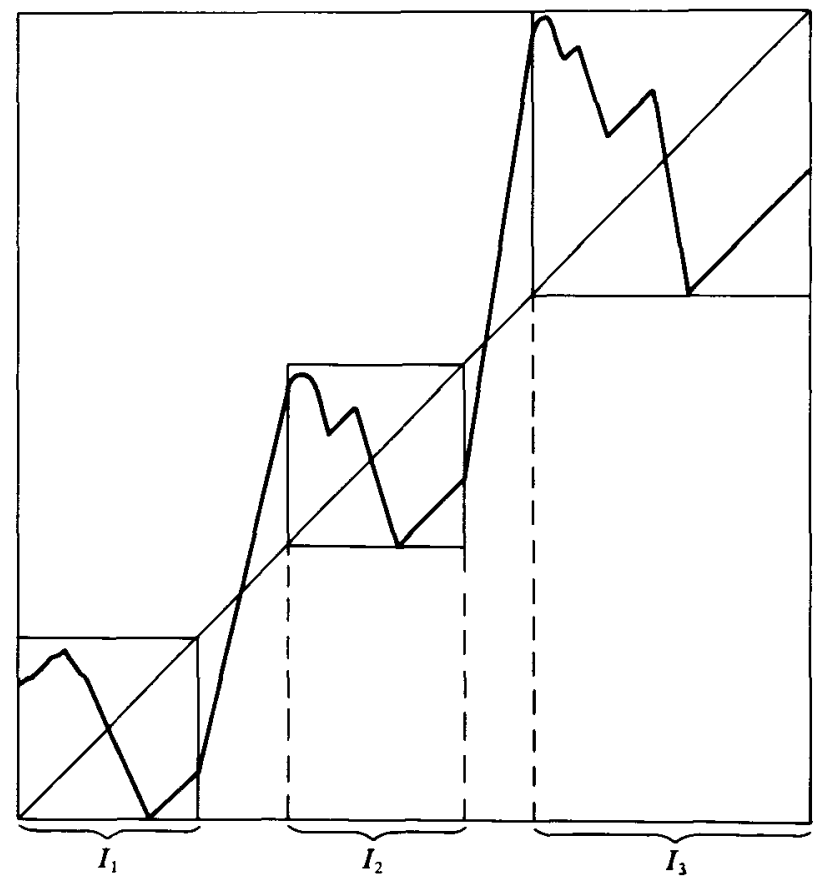

FIGURE 7

Suppose we wish to construct a map $f$ with an infinite set of strict containments in (*), occurring after the sets $\Omega\left(f^{2 n}\right)$ for $n=n_{1}, n_{2}, \ldots$ Choose the intervals $I_{1}, I_{2}, \ldots$ so that the sum of their lengths and the lengths of the intervals between them converges. (This can be done by 'scaling down' the intervals on which the maps $f_{n_{k}}$ are defined.) Let $g_{m}: J_{m} \rightarrow J_{m}$ be the map defined in the preceding paragraph for the finite set consisting of the first $m$ strict containments. Then $J_{1} \subseteq J_{2} \subseteq \cdots$. Let $J=\operatorname{cl}\left[\bigcup J_{m}\right]$ 
and extend each $g_{m}$ to all of $J$ by letting the right-hand endpoint of $J$ be a fixed point and defining $g_{m}$ linearly between the right-hand endpoints of $J_{m}$ and $J$. Then the sequence of functions $\left\{g_{m}\right\}$ converges uniformly to a function $f$ which has the required sequence of strict containments in (*) (figure 8 ).

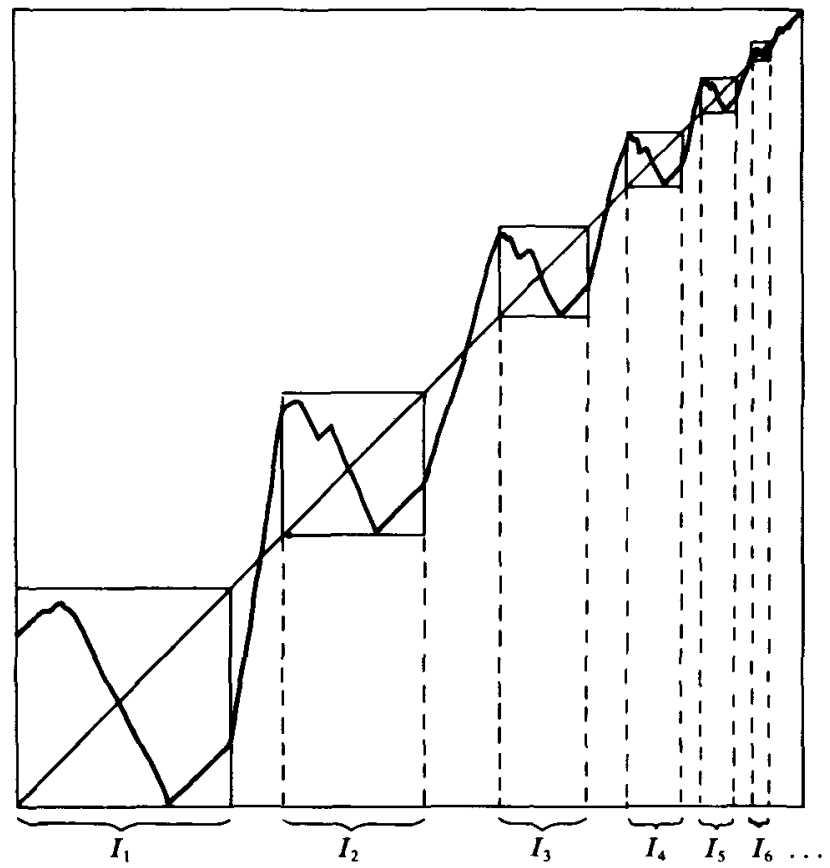

Figure 8

The reader will have observed that Štefan's construction creates a 'corner' at $x=2$ (and possibly one at $x=1$ as well), thus destroying whatever smoothness the original map possessed. However, this corner can be smoothed if we allow $J$ to be somewhat larger, say $J=[-\varepsilon, 3]$, and define $g$ as in figure 9. (A similar construction will smooth a corner at $x=1$.) This can be done without creating additional non-wandering points.

We can use the smooth version of Štefan's construction to construct smooth examples for any finite set of strict containments in (*). If there are infinitely many strict containments in $(*)$, then this procedure yields a map which is neither piecewise monotone nor smooth, although it fails to be smooth only at the right-hand endpoint.

\section{Maps of higher-dimensional spaces}

In this section, we briefly examine to what extent our results carry over to maps of higher-dimensional spaces. K. Sawada [12] has recently constructed an example of a diffeomorphism $g$ of the 2 -sphere for which $\Omega(g) \neq \Omega\left(g^{2}\right)$. This example leaves open the possibility that the conclusion of our main theorem $\left(\Omega(g)=\Omega\left(g^{n}\right)\right.$ for all odd $n \geq 1$ ) holds for maps of compact manifolds of dimension greater than one. 

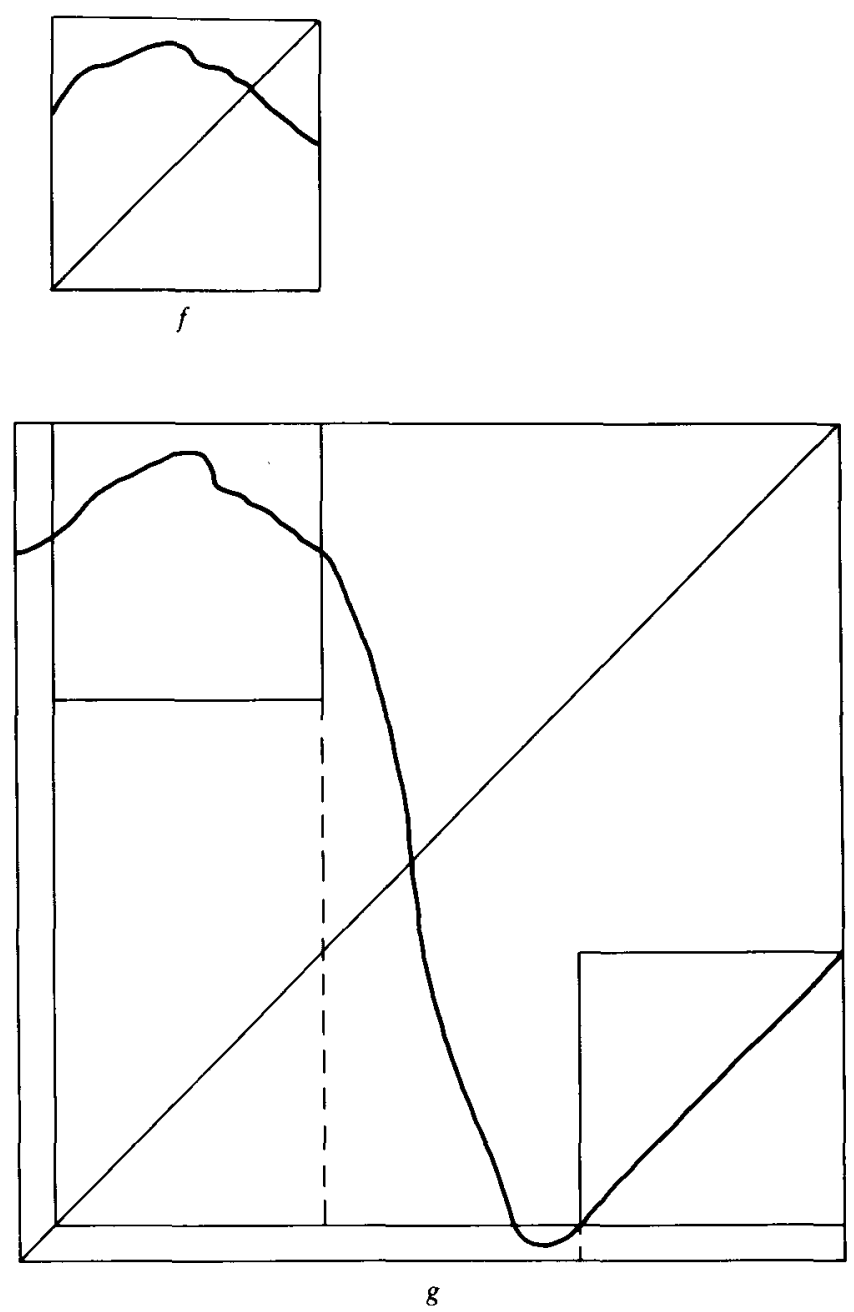

FIGURE 9

We shall show that for any $n \geq 2$, there is an orientation-preserving diffeomorphism $g$ of the closed 2-disk such that $\Omega(g) \neq \Omega\left(g^{n}\right)$. Such a diffeomorphism can be extended to any compact surface and then to any compact manifold of dimension greater than one.

Consider a flow on the square $[0,1] \times[0,1]$ with the orbit picture shown in figure 10. Each edge is invariant. There are eight fixed points, one at each corner and four others $-a_{i}=(i, a)$ and $b_{i}=(i, b)(i=0,1)-$ lying on the vertical edges. These last four fixed points are of saddle-node type. We assume for definiteness that $a_{i}$ lies above $b_{i}$. For example, define the flow near $a_{i}$ and $b_{i}$ by

$$
\begin{aligned}
& \dot{x}_{1}=\left(x_{1}-i\right)^{2} \\
& \dot{x}_{2}= \begin{cases}a-x_{2} & \text { near } a_{i} \\
x_{2}-b & \text { near } b_{i} .\end{cases}
\end{aligned}
$$




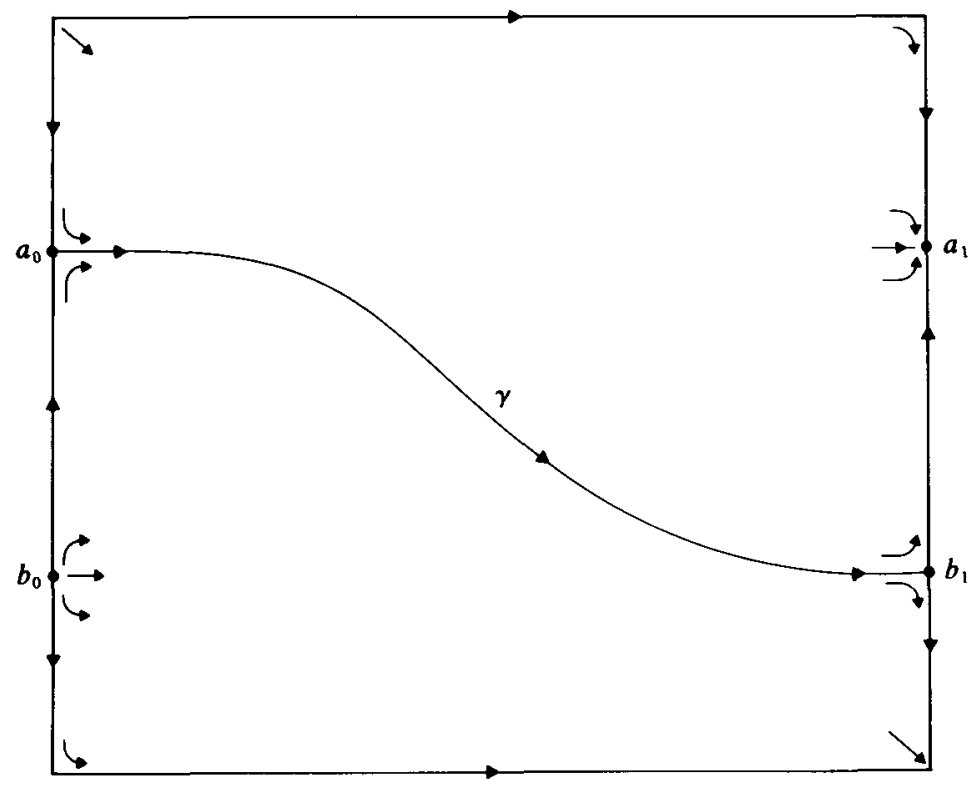

Figure 10

Then there is a unique orbit with $\alpha$-limit set $\left\{a_{0}\right\}$ and a unique orbit with $\omega$-limit set $\left\{b_{1}\right\}$. We assume these orbits are the same saddle-connection orbit $\gamma$. Finally, we assume that

$$
\dot{x}_{1}>0 \text { for } 0<x_{1}<1 \text {. }
$$

The time-one map of such a flow can be modified near some point of $\gamma$ so that for the resulting diffeomorphism $\psi, W^{u}\left(a_{0}, \psi\right)$ intersects $W^{s}\left(b_{1}, \psi\right)$ transversally (figure 11). (See, for example, [10, pp. 207-208].)

Then [10, p. 192],

(1) $\operatorname{cl}\left[W^{s}\left(b_{1}, \psi\right)\right] \supseteq W^{u}\left(a_{0}, \psi\right)$.

Since the invariant manifolds of a fixed point of a map are the same for any positive power of the map, we may, for any $n \geq 2$, replace $\psi$ with $\psi^{n}$ in (1). In particular, (2) For any $n \geq 2$ and any $x=(0, c)$ and $y=(1, d)$ with $c$ and $d$ between $a$ and $b$, there exist $x_{i} \rightarrow x$ and $k_{i} \rightarrow+\infty$ such that $\psi^{k_{i} n}\left(x_{i}\right) \rightarrow y$.

Now glue together $n$ copies of the square (identifying $a_{0}$ in each cell with $a_{1}$ in the next cell in the clockwise direction, etc.) to form an annulus, and extend $\psi$ to the annulus in the obvious way. Let $\tau$ be an orientation-preserving diffeomorphism of the annulus, commuting with $\psi$, which translates each cell clockwise by one cell. Let $g=\psi \circ \tau$. Finally, extend $g$ to the (small) disk inside the annulus, so as to obtain an orientation-preserving diffeomorphism of the (large) disk (figure 12).

(11.1) Proposition. With notation as above, $\Omega(g) \neq \Omega\left(g^{n}\right)$.

Proof. On the annulus, $g^{n}=\psi^{n}$. Therefore any point of $\Omega\left(g^{n}\right)$ which is an interior point of the annulus must also be in $\Omega\left(\psi^{n}\right) \subseteq \Omega(\psi)$. But it is easy to see that $\Omega(\psi)$ contains only the fixed points of $\psi$. 


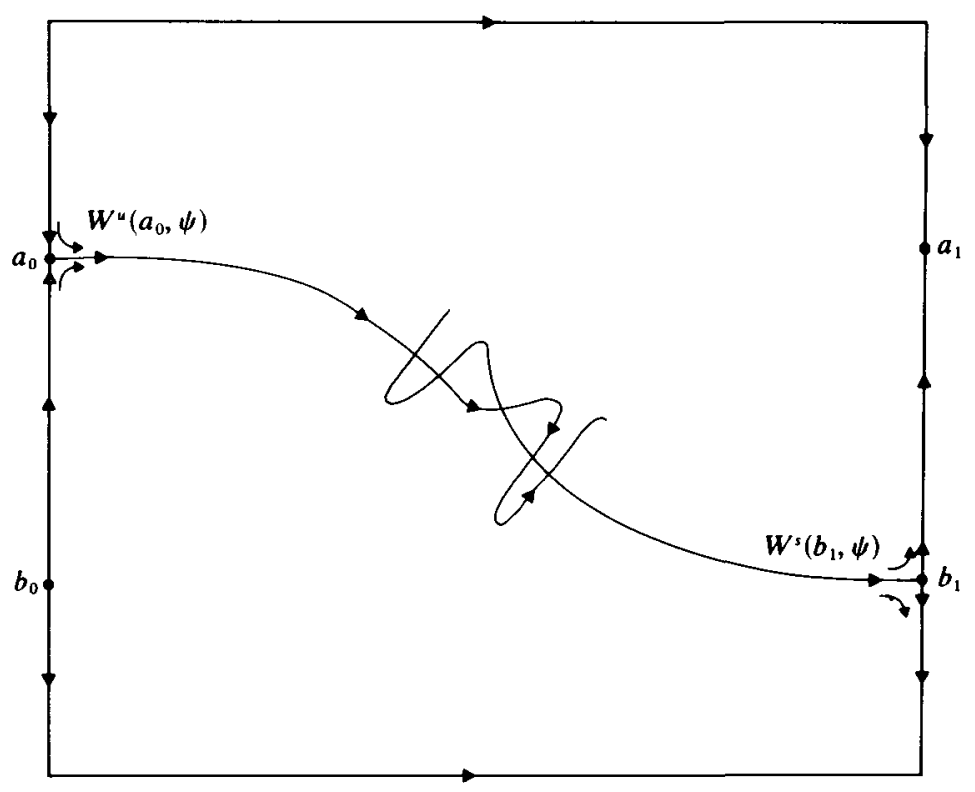

FIGURE 11

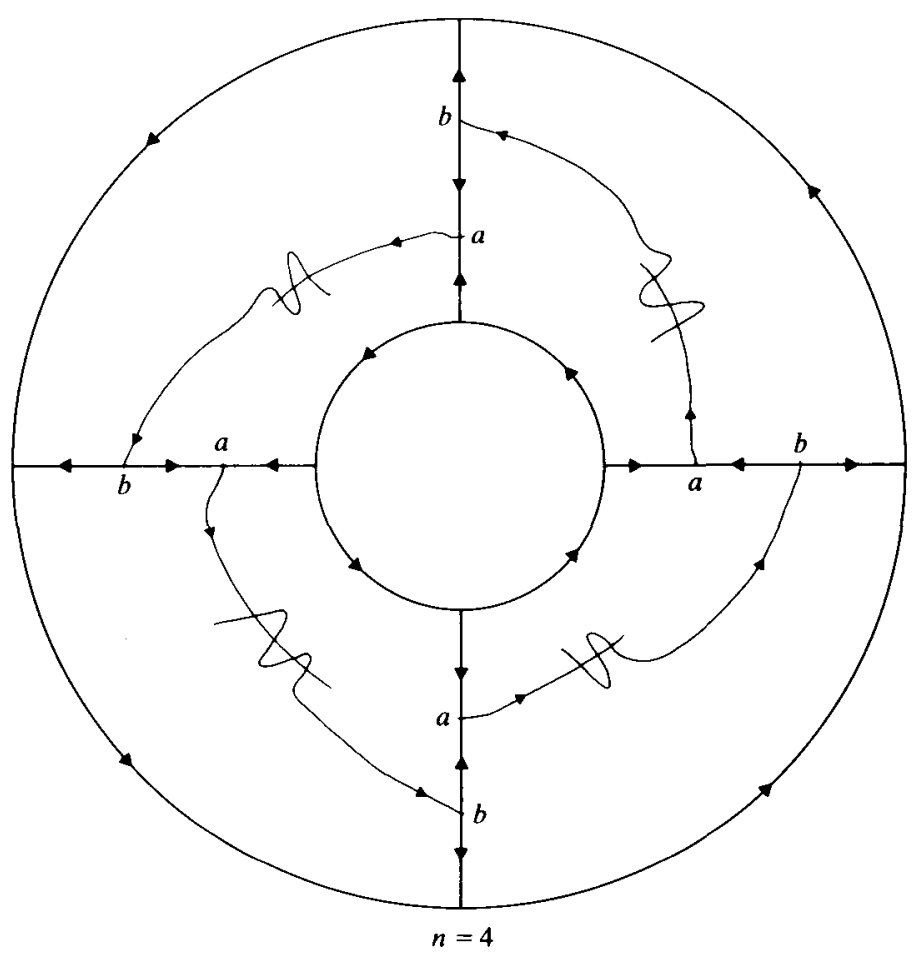

FIGURE 12 
We show that every point lying between $a$ and $b$ on a radial segment is in $\Omega(g)$. Let $x$ be such a point. Then $g^{-1}(x)$ lies between $a$ and $b$ on the next radial segment in the counter-clockwise direction. Since $g^{n}=\psi^{n}$ on the annulus, by (2) there exist $x_{i} \rightarrow x$ and $k_{i} \rightarrow+\infty$ such that $g^{k_{i} n}\left(x_{i}\right) \rightarrow g^{-1}(x)$. Then $g^{k_{i} n+1}\left(x_{i}\right) \rightarrow x$ and hence $x \in \Omega(g)$.

The argument used in the proof of (11.1) can be used to show that $\Omega(g) \neq \Omega\left(g^{k}\right)$ unless $k$ and $n$ are relatively prime. Is this a general phenomenon? More specifically, if $g: M \rightarrow M$ where $\operatorname{dim} M \geq 2$ and if $x \in \Omega(g)-\Omega\left(g^{n}\right)$, then must there exist $m=m(x)$ such that $x \in \Omega\left(g^{k}\right)$ if and only if $k$ and $m$ are relatively prime? Cf. (9.1).

\section{REFERENCES}

[1] J. Auslander \& P. Siebert. Prolongations and stability in dynamical systems. Ann. Inst. Fourier (Grenoble) 14 (1964), 237-267.

[2] N. P. Bhatia \& G. P. Szegö. Dynamical Systems: Stability Theory and Applications. Springer Lecture Notes in Math. no. 35. Springer: Berlin, 1967.

[3] L. Block. Homoclinic points of mappings of the interval. Proc. Amer. Math. Soc. 72 (1978), 576-580.

[4] E. M. Coven \& G. A. Hedlund. $\bar{P}=\bar{R}$ for maps of the interval. Proc. Amer. Math. Soc. 79 (1980), 316-318.

[5] A. Denjoy. Sur les courbes définies par les équations différentielles à la surface du tore. J. Math. Pures Appl. 11 (1932), 333-375.

[6] P. Erdös \& A. H. Stone. Some remarks about almost periodic transformations. Bull. Amer. Math. Soc. 51 (1945), 126-130.

[7] W. H. Gottschalk. Powers of homeomorphisms with almost periodic properties. Bull. Amer. Math. Soc. 50 (1944), 222-227.

[8] J. Guckenheimer. Sensitive dependence to initial conditions for one dimensional maps. Comm. Math. Phys. 70 (1979), 133-160.

[9] M. Misiurewicz. Absolutely continuous measures for certain maps of the interval. I.H.E.S. preprint, 1979.

[10] Z. Nitecki. Differentiable Dynamics: An Introduction to the Orbit Structure of Diffeomorphisms. M.I.T. Press: Cambridge, 1971.

[11] Z. Nitecki. Periodic and limit orbits and the depth of the center for piecewise monotone interval maps. Proc. Amer. Math. Soc. 80 (1980), 511-514.

[12] K. Sawada. On the iteration of diffeomorphisms without $C^{0} \Omega$-explosions. Proc. Amer. Math. Soc. 79 (1980), 110-112.

[13] P. Štefan. A theorem of Šarkovskii on the existence of periodic orbits of continuous endomorphisms of the real line. Comm. Math. Phys. 54 (1977), 237-248.

[14] T. Ura. Sur les courbes définies par les équations différentielles dans l'espace à $m$ dimensions. Ann. Sci. École Norm. Sup. 70 (1953), 287-360.

[15] L.-S. Young. A closing lemma on the interval. Invent. Math. 54 (1979), 179-187. 